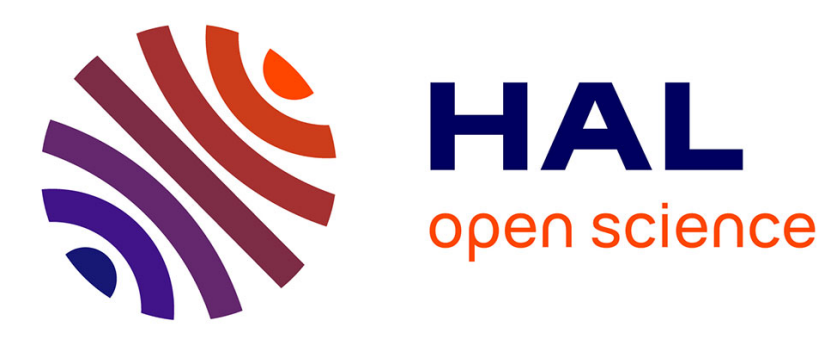

\title{
L'instrumentation du contrôle de gestion dans les entreprises au Sénégal
}

Boniface Bampoky, François Meyssonnier

\section{To cite this version:}

Boniface Bampoky, François Meyssonnier. L'instrumentation du contrôle de gestion dans les entreprises au Sénégal. Comptabilités et innovation, May 2012, Grenoble, France. pp.cd-rom. hal00690927

\section{HAL Id: hal-00690927 \\ https://hal.science/hal-00690927}

Submitted on 24 Apr 2012

HAL is a multi-disciplinary open access archive for the deposit and dissemination of scientific research documents, whether they are published or not. The documents may come from teaching and research institutions in France or abroad, or from public or private research centers.
L'archive ouverte pluridisciplinaire HAL, est destinée au dépôt et à la diffusion de documents scientifiques de niveau recherche, publiés ou non, émanant des établissements d'enseignement et de recherche français ou étrangers, des laboratoires publics ou privés. 


\section{L'instrumentation du contrôle de gestion dans les entreprises au Sénégal}

Boniface BAMPOKY* et François MEYSSONNIER**

\begin{abstract}
Résumé
Des entretiens exploratoires auprès de 10 entreprises suivis d'une enquête confirmatoire par questionnaires auprès de 130 entreprises représentatives permettent de décrire les pratiques de contrôle de gestion des entreprises au Sénégal.

\begin{tabular}{|c|c|c|}
\hline $\begin{array}{l}\text { MOTS-CLES : } \\
\text { BUDGETS - T }\end{array}$ & $\begin{array}{l}\text { CALC } \\
\text { ABL }\end{array}$ & $\begin{array}{l}\text { ULS DE } \\
\text { EAUX D }\end{array}$ \\
\hline PETITES & ET & MC \\
\hline $\begin{array}{l}\text { ENTREPRISES } \\
\text { NATIONALES. }\end{array}$ & - & FIRMES \\
\hline
\end{tabular}
\end{abstract}

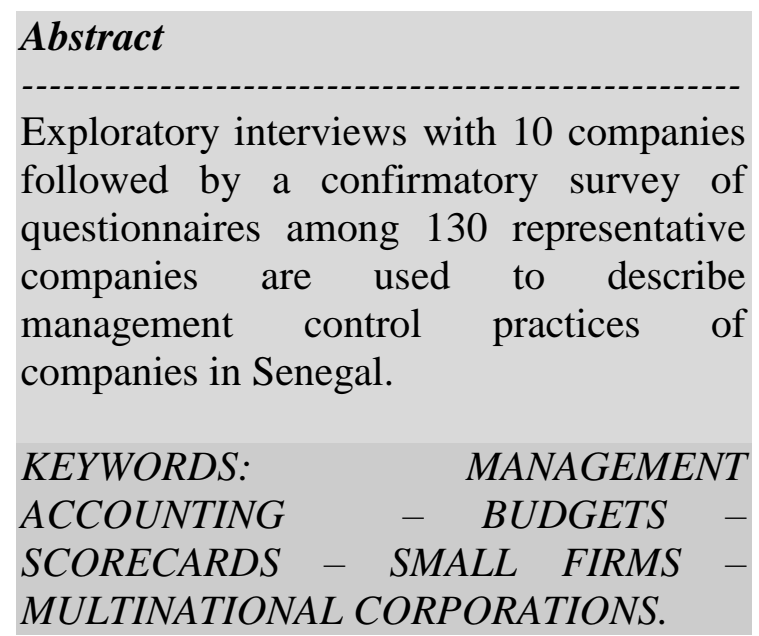

* Assistant à l'Université Cheikh Anta Diop de Dakar (Sénégal)

** Professeur à l'Université de Nantes (France)

\section{Correspondance :}

Boniface Bampoky

Ecole Supérieure Polytechnique

(Université Cheikh Anta Diop de Dakar)

BP 15839 Dakar-Fann

Dakar

Sénégal

bbampoky@hotmail.com
François Meyssonnier

IEMN-IAE

(Université de Nantes)

Chemin de la Censive du Tertre

44000 Nantes

France

francois.meyssonnier@univ-nantes.fr 


\section{Introduction}

Les pratiques de contrôle de gestion dans les pays développés sont l'objet d'études depuis longtemps. On a maintenant une connaissance assez complète des méthodes et outils de contrôle de gestion utilisés dans les entreprises françaises, européennes, japonaises ou nordaméricaines. Par contre, dans les pays du Sud en voie de développement, les recherches sont beaucoup plus réduites. Si des travaux de recherche commencent à apparaître sur l'instrumentation du contrôle de gestion dans les pays émergents d'Asie, d'Amérique latine ou plus près de nous, du Maghreb, il y en a beaucoup moins pour les pays les moins avancés par exemple en Afrique noire au sud du Sahara, si on met de côté l'Afrique du Sud dont le niveau de développement réel (hors rente liée à l'exploitation des matières premières) est bien supérieur à celui des autres pays de la zone. C'est pourquoi nous avons décidé de réaliser une étude descriptive des démarches et outils du contrôle de gestion mis en œuvre au Sénégal. Il s'agit de la première étude de ce type réalisée dans ce pays et, à notre connaissance, une des rares réalisées dans un pays d'Afrique de l'Ouest avec celle de Ngongang (2010) portant sur les PMI du Cameroun. Ce travail à pour objectif de mieux connaître la réalité des entreprises des pays du Sud en matière d'instrumentation du contrôle de gestion afin d'en déduire les évolutions prévisibles ou souhaitables pour accroître l'efficience et l'efficacité de la gestion des entreprises (spécifiquement dans le cas des entreprises familiales qui représentent un enjeu crucial pour la croissance de ces pays).

Dans notre démarche, nous avons privilégié une vision rationaliste et instrumentale du contrôle de gestion par rapport à une approche focalisée sur les aspects sociologiques et sur les cultures nationales ou ethniques. Les apports de l'ethnicité à la compréhension des outils et pratiques de contrôle de gestion (Joannides, 2011) nous semblent très utiles et complémentaires de l'approche technique mais ne sont pas la porte d'entrée première quand on veut appréhender la réalité de l'outillage du contrôle de gestion. En effet contrairement à cet auteur, nous trouvons trop vaste une définition du contrôle de gestion par : "l'ensemble des dispositifs intentionnels ou non portant sur l'organisation, le groupe social, le pouvoir d'une personne, l'individu contrôlé lui-même » ou une définition des sciences de gestion comme «toutes les disciplines appréhendant la vie des organisations». Nous avons une vision beaucoup plus modeste du contrôle de gestion. Pour nous il est important de bien distinguer trois choses: le contrôle organisationnel qui est l'ensemble des éléments, (intentionnels ou non, formalisés ou pas) qui permettent à une organisation, quelque soit sa nature (église, Etat, entreprise, mafia, etc.) et son contexte (niveau de développement de l'économie, cultures nationales, etc.), de «tenir ensemble »; les systèmes de pilotage de la performance (SPP) qui sont la mise en œuvre cohérente de dispositifs, intentionnels et formalisés, qui visent à la réalisation des objectifs de l'entreprise (avec des aspects stratégiques, le système d'information comptable qui est central et la dimension RH, entre autres); le contrôle de gestion enfin qui est au centre du SPP et regroupe les outils et méthodes visant à améliorer la qualité des choix de gestion et à assurer la convergence des comportements (comptabilité de gestion, budgets, tableaux de bord, systèmes d'information, structuration de l'audit interne).

Au Sénégal, le développement économique est très faible. Le PNB par habitant est de 708 \$ seulement (contre 1694 \$ par habitant au Maroc et 35854 \$ par habitant en France) et l'économie souterraine et les activités informelles y sont importantes. Nous avons choisi de nous intéresser aux entreprises déclarées et reconnues, moyennes ou grandes (laissant de côté les activités informelles et les petites entreprises de moins de 50 salariés où l'instrumentation du contrôle de gestion est de toutes façons très réduite partout dans le monde). Le terrain de 
recherche est donc celui des filiales de firmes multinationales installées au Sénégal, des entreprises publiques ou parapubliques (comme beaucoup d'autres pays africains, le Sénégal est dans une période de privatisation de nombreuses entreprises contrôlées jusque-là par l'Etat) et des entreprises locales moyennes (familiales ou non). Nous souhaitons décrire et expliquer l'état des pratiques en matière de pilotage de la performance. Pour cela nous allons procéder de façon tout à fait classique. Un état de l'art va être effectué (1). La problématique et la méthodologie vont être explicitées (2). Une étude qualitative exploratoire va être réalisée (3). Une enquête quantitative confirmatoire suivra (4). Les résultats seront discutés (5). Ce qui nous permettra de conclure sur les apports et les limites de ce travail de recherche.

\section{Etat de l'art}

Si on s'intéresse au contrôle de gestion dans les pays en développement, on est au croisement de différents champs de la littérature en contrôle de gestion.

Il faut d'abord prendre en compte le contrôle de gestion dans sa globalité. Les concepts et outils du contrôle de gestion ont été développés par de nombreux auteurs. Anthony (1965) et Anthony et Govindarajan (1998) présentent comment les méthodes, les outils et les comportements s'articulent dans les dispositifs de contrôle de gestion. Bouquin (2008) a précisé le cadre du contrôle de gestion avec son analyse des phases de planification, pilotage et post-évaluation. Kaplan et Norton (2001) ont promu le développement des indicateurs de pilotage au travers d'un tableau de bord stratégique d'ensemble, le balanced scorecard. Simons (1995) propose une vision globale des systèmes de contrôle intégrant 4 leviers : les croyances, les barrières, le contrôle de gestion diagnostique et le contrôle de gestion interactif. Tout ceci trouve sa place dans le cadre des systèmes unifiés de pilotage de la performance décrits notamment par Ferreira et Otley (2009). Ces approches permettent de bien comprendre l'usage d'outils (dont la rationalité propre doit être discutée) activés par des acteurs qui se les approprient dans un contexte (dimensions psychocognitive et sociopolitique) afin de réaliser les missions qui sont les leurs dans l'entreprise : le calcul et l'analyse des coûts ; la gestion conjointe du triplet coûts-qualité-délais; l'optimisation de la création de valeur pour l'actionnaire ; la mise sous tension de l'organisation ; etc.

Ensuite, bien entendu, il y a toute la littérature classique de référence en matière d'instrumentation du contrôle de gestion. On sait beaucoup de choses et de façon assez détaillée sur les pratiques budgétaires et leurs aspects contingents (Berland, 2002 ; Lambert et Sponem, 2009). Il en est de même pour les techniques de calculs de coûts, d'établissement des devis, de mise en évidence des marges ou des résultats en fonction de la taille des entreprises et des métiers (Nobre, 2001 ; Mévellec, 2005 ; Ngongang, 2010). Par contre, sur certains outils, les résultats sont divergents ou sujets à polémiques. Ainsi la méthode UVA fondée sur les équivalents de production a probablement une utilisation cantonnée à un nombre très réduit d'entreprises (de La Villarmois et Levant, 2010) malgré la place assez importante que lui consacrent la littérature académique et les manuels en France. De même la méthode de calculs de coûts $\mathrm{ABC}$ est à l'évidence bien moins répandue que ce qu'affirment certaines études sur la diffusion de cette innovation managériale dans le monde avec souvent d'ailleurs des résultats contradictoires (Gosselin et Mévellec, 2003). Des travaux se développent par ailleurs sur les méthodes d'action sur les coûts pour les réduire, à travers par exemple les démarches de target costing (Meyssonnier, 2001). On s'intéresse de plus en plus aux pratiques de contrôle inter-organisationnel que ce soit dans le cadre des relations clientfournisseurs (Donada et Nogatchewsky, 2008) ou des réseaux de franchise (Goullet et Meyssonnier, 2011). L'usage du balanced scorecard se répand aussi dans les entreprises mais 
avec des objectifs qui peuvent être assez différents. C'est un outil de communication vis à vis des parties prenantes ou un outil de représentation stratégique ou enfin réellement un instrument de pilotage de la performance complémentaire ou alternatif aux budgets (Choffel et Meyssonnier, 2005). Le contrôle de gestion sociétal, permettant de répondre aux besoins des démarches de responsabilité sociale et environnementale des entreprises, se développe et est également étudié par les chercheurs (Meyssonnier et Rasolofo-Distler, 2008). Enfin plus récemment des travaux émergent sur la dimension opérationnelle des pratiques de contrôle dans les activités de service (Meyssonnier, 2011). A partir des recherches de ce type, orientées sur la dimension instrumentale du contrôle de gestion, les chercheurs sont capables, en réponse à la demande sociale, de proposer des instruments de gestion plus adaptés aux besoins des entreprises et d'améliorer l'outillage de gestion en vue de plus d'efficacité. Ils peuvent aussi développer des points de vue réflexifs voire critiques sur les aspects sociaux et politiques des pratiques de contrôle (Bourguignon, 2009).

La connaissance et l'usage des outils de gestion modernes par les entreprises des pays les moins avancés nécessite de se pencher sur la question des modes de transmission des connaissances en gestion et des artefacts supports (outils techniques ou méthodes intellectuelles) de gestion. Les facteurs de diffusion des techniques et outils du contrôle de gestion ont été étudiés par Abrahamson (1991) et Rogers (1995) qui présentent des analyses des raisons de l'adoption ou du rejet des innovations managériales. Les difficultés de la structuration de la gestion en PME sont mises en évidence dans une étude de cas par Meyssonnier et Zawadzki (2008). Alcouffe, Berland et Levant (2003) comparent les modes de diffusions de trois innovations en France : les budgets, la méthode UVA, la méthode ABC. Moalla (2007) étudie le développement de la méthode ABC au Maroc. Toutefois on doit remarquer que la plupart de ces auteurs accordent plus d'importance aux facteurs institutionnels et aux dimensions communicationnelles et sociologiques qu'aux caractéristiques intrinsèques des outils qui expliquent pourtant aussi nous semble-t-il, et de façon non négligeable, le succès ou l'échec de leur adoption (cf. dans le cas de la méthode UVA la critique technique de l'outil par Meyssonnier en 2003).

L'impact des cultures nationales sur le contrôle de gestion est également essentiel à la compréhension de l'usage de l'instrumentation de gestion conçue dans les entreprises les plus innovantes. La diversité des comportements en entreprise selon les pays a été étudiée par Hofstede (1980) dans ses travaux analytiques sur la diversité culturelle et la mise en évidence des principaux traits distinguant les cultures nationales: individualisme/collectivisme; distance hiérarchique; aversion au risque ; masculinité/féminité ; horizon temporel. D’Hiribarne (1989) a aussi étudié les systèmes de contrôle organisationnels (mais dans une approche holiste) et a dégagé une grille de lecture opposant la logique de l'honneur en France, la logique du contrat aux Etats-Unis et la logique du consensus aux Pays-Bas. De nombreuses recherches ont été aussi développées sur les systèmes de contrôle de gestion des firmes multinationales. On distingue alors les firmes globales dont la stratégie est fondée sur l'intégration au niveau mondial et les économies d'échelle et de gamme, d'une part, et les firmes multidomestiques s'adaptant aux spécificités des marchés locaux et des réglementations nationales, d'autre part. Dans le premier cas les systèmes de contrôle de gestion seront centralisés et standardisés, dans le second ils seront plus autonomes et différenciés en fonction des contextes. Tout ceci a été analysé par Mouritsen (1995) ou Quattrone et Hopper (2005) et de nombreuses études de cas ont été présentées par Wickramasinghe, Hopper et Rathnasiri (2004), Busco, Giovannoni et Riccaboni (2007) ou Efferin et Hopper (2007). Au Sénégal, peu intégré dans les chaînes de 
valeur internationales, on a pour le moment affaire essentiellement à des firmes multinationales de type multidomestiques.

Plusieurs auteurs se sont intéressés aux modes de contrôle des organisations locales et plus particulièrement, par rapport à notre problématique, en Afrique. Les modes de gestion des entreprises du secteur informel en Afrique ont été analysés par Hernandez (1993, 1999). Des exemples de réussites de PME sénégalaises sont présentés par Bellot (1988). Les caractéristiques de la gestion des PME familiales ont été présentées dans un contexte tunisien par Mzid Ben Amar et Mezghani (2010). La spécificité des entreprises familiales africaines est décrite par Boungou Bazika (2005). Wade (2003) a étudié l'introduction du contrôle de gestion en milieu hospitalier au Sénégal. Le rôle de l'ethnicité a été souligné par Kamdem et Ongodo (2004). Gervais et Kaboré (2007) ont insisté sur les dimensions sociétales à prendre en compte dans l'approche des pratiques de gestion au Burkina-Fasso. Les spécificités de la gestion dans le monde islamique ont été étudiées, sur la base d'études de cas par d'Iribrane (2007) et Yousfi (2007) ou à partir d'enquête par Benabdeljlil (2007). L'existence d'une culture européenne en comptabilité et contrôle de gestion a aussi été discutée par Löning (1995).

Nous avons donc là un ensemble de travaux qui devraient nous permettre d'appréhender conceptuellement la réalité des pratiques de contrôle de gestion dans les entreprises au Sénégal.

\section{Problématique et méthodologie de la recherche}

Nous avons fait le choix de centrer le travail sur la dimension instrumentale du contrôle de gestion et de réaliser une approche fondée sur des entretiens et un questionnaire diffusé très largement. Il nous semble en effet que les instruments de gestion, fondés sur des méthodes intellectuelles et des technologies de l'information, structurent largement les processus humains en œuvre dans les entreprises. Ceci est vrai aussi bien pour un pays peu développé comme le Sénégal que pour les pays avancés, pour peu qu'on s'intéresse aux entreprises modernes et formalisées qui correspondent à l'objet de notre étude. Par ailleurs, si nous avions décidé de travailler prioritairement sur la dimension comportementale, le travail d'observation in situ n'aurait pu avoir lieu que sur un nombre très limité d'entreprises et sous la forme d'études de cas longitudinales. Son pouvoir de généralisation aurait été réduit. Nous avons bien conscience que l'insertion sociale et les questions d'usage des outils de gestion sont cruciales, encore faut-il savoir d'abord si ces outils sont présents et sous quelle forme dans les entreprises du Sénégal. La question de l'existence des outils précède la question de l'usage de ces mêmes outils.

La question principale retenue dans notre recherche est donc la suivante : "Quels sont les méthodes et outils de contrôle de gestion dans les entreprises au Sénégal ? ». Elle correspond à une approche fondamentalement descriptive. Nous nous sommes intéressés aux entreprises formalisées de plus de 50 salariés. La population étudiée est celle des entreprises moyennes ou grandes immatriculées au registre du commerce, enregistrées à l'Agence Nationale de la Statistique et de la Démographie du Sénégal, payant des impôts et déclarant leurs employés. Ces entreprises sont de trois catégories : les filiales locales des firmes multinationales $(20 \%$ des entreprises de plus de 50 salariés officiellement déclarées au Sénégal), les entreprises publiques ou parapubliques (15\%) et les entreprises privées familiales ou non (65\%). 
Dans une phase préalable de cadrage de l'étude, des contacts, échanges et discussions ont eu lieu avec des représentants des principales organisations patronales et professionnelles (dans le domaine de la comptabilité et du contrôle) du Sénégal : le Club des Investisseurs Français $\mathrm{Au}$ Sénégal (CIFAS), l'Association des Femmes d'Affaires et Femmes Commerçantes (AFAC), la Confédération Nationale des Employeurs du Sénégal (CNES), le Conseil National du Patronat du Sénégal (CNPS), l'Ordre National des Experts Comptables et Comptables Agréés (ONECCA). Ces échanges avec des professionnels de référence, experts de la gestion des entreprises ou du système comptable et du contrôle de gestion, nous ont permis de mettre en évidence les principales questions et les enjeux de l'étude ainsi que de cerner les traits essentiels de la réalité du contrôle de gestion au Sénégal. Ensuite une phase exploratoire a eu lieu, sur la période 2008-2009, avec des entretiens dans une dizaine d'entreprises dans cinq secteurs économiques significatifs du Sénégal. Ceci a permis de dégager des hypothèses sur l'état de développement et les caractéristiques de l'instrumentation du contrôle de gestion dans les entreprises au Sénégal. Un questionnaire a ensuite été construit et testé. Une phase confirmatoire a eu lieu, sur la période 2009-2010, avec l'administration puis l'exploitation statistique du questionnaire auquel ont répondu 130 entreprises représentatives du tissu économique du pays.

\section{Etude qualitative exploratoire}

L'étude exploratoire par entretiens en vis-à-vis a été réalisées auprès de dix entreprises choisies par paires dans cinq secteurs clés: l'immobilier, l'agro-alimentaire, les télécommunications, la savonnerie, les banques (cf. tableau 1 ci-dessous). Les entretiens semi directifs ont été effectués avec les cadres responsables à l'aide d'un guide d'entretien (cf. annexe 1). Le maximum de documents a été collecté sur place. Un compte-rendu a été réalisé pour chaque entretien et éventuellement des informations complémentaires ont été demandées ex post. La base de travail a été un échantillon de convenance. La collecte d'information s'est arrêtée quand il nous a semblé que la multiplication d'entretiens ne changerait pas la vision qui ressortait de cette première phase (principe de saturation). 
Tableau 1 : Les 10 entreprises retenues pour les entretiens

\begin{tabular}{|c|c|c|c|}
\hline Entreprise & Secteur & Taille et nationalité & $\begin{array}{c}\text { Fonction de } \\
\text { l'interlocuteur }\end{array}$ \\
\hline $\begin{array}{l}\text { Société Nationale } \\
\text { des Habitations à Loyer } \\
\text { Modéré (SN-HLM) }\end{array}$ & \multirow{2}{*}{$\begin{array}{l}\text { Aménagement } \\
\text { immobilier }\end{array}$} & $\begin{array}{l}\text { Entreprise sénégalaise } \\
\text { publique (180 salariés) de } \\
\text { logement social }\end{array}$ & Contrôleur de Gestion \\
\hline $\begin{array}{l}\text { Société Immobilière du } \\
\text { Cap-Vert (SICAP) }\end{array}$ & & $\begin{array}{l}\text { Entreprise sénégalaise } \\
\text { parapublique ( } 80 \text { salariés) } \\
\text { à prix de vente libres }\end{array}$ & Contrôleur de Gestion \\
\hline $\begin{array}{l}\text { Savonnerie Africaine } \\
\text { Fakhry (SAF) }\end{array}$ & \multirow[b]{2}{*}{ Savonnerie } & $\begin{array}{l}\text { Entreprise créée par une } \\
\text { famille libano- } \\
\text { sénégalaise (110 salariés) }\end{array}$ & Chef Comptable \\
\hline $\begin{array}{l}\text { Savons « Le Marabout » } \\
\text { (Alminco) }\end{array}$ & & $\begin{array}{l}\text { Entreprise sénégalaise } \\
\text { (110 employés) issue } \\
\text { d'une partition de } \\
\text { Colgate Palmolive } \\
\text { Sénégal }\end{array}$ & Chef du Personnel \\
\hline Orange (SONATEL) & \multirow{2}{*}{ Téléphonie Mobile } & $\begin{array}{l}\text { Une des plus grandes } \\
\text { entreprise au Sénégal, } \\
\text { filiale d'un groupe } \\
\text { français (plus de } 2000 \\
\text { employés) }\end{array}$ & Contrôleur de Gestion \\
\hline Tigo & & $\begin{array}{l}\text { Filiale d'un groupe } \\
\text { luxembourgeois ( } 300 \\
\text { salariés) }\end{array}$ & Chef Comptable \\
\hline Nestlé Sénégal & \multirow{2}{*}{ Agro-Alimentaire } & $\begin{array}{l}\text { Filiale (330 salariés) } \\
\text { d'une grande entreprise } \\
\text { suisse }\end{array}$ & Contrôleur de Gestion \\
\hline SATREC & & $\begin{array}{l}\text { Entreprise sénégalaise } \\
\text { (120 salariés) }\end{array}$ & Directeur Financier \\
\hline $\begin{array}{l}\text { Compagnie Bancaire de } \\
\text { l'Afrique de l'Ouest } \\
\text { (CBAO) }\end{array}$ & \multirow{2}{*}{ Banque } & $\begin{array}{l}\text { Filiale sénégalaise (plus } \\
\text { de } 1000 \text { salariés) de la } \\
\text { première banque } \\
\text { marocaine (Attijari Wafa) }\end{array}$ & Contrôleur de Gestion \\
\hline $\begin{array}{l}\text { Crédit Agricole Sénégal } \\
\text { (CAS) }\end{array}$ & & $\begin{array}{l}\text { Ancienne filiale (210 } \\
\text { salariés) du Crédit } \\
\text { Agricole de France } \\
\text { récemment rachetée par } \\
\text { Attijari Wafa }\end{array}$ & $\begin{array}{l}\text { Directeur Finances et } \\
\text { Comptabilité }\end{array}$ \\
\hline
\end{tabular}


Dans les entreprises publiques ou parapubliques du secteur de l'aménagement immobilier, le poids des jeux clientélistes est lourd et oblige à ruser pour tenter d'y résister : "Des politiques nous imposent souvent des agents. C'est le danger. Il nous arrive alors de faire appel à des consultants pour définir plus objectivement les besoins réels. » (contrôleur de gestion SNHLM) ou encore «Après constatation de l'impact négatif des contraintes que nous imposaient les politiques, nous avons procédé à une rationalisation des procédures de recrutement. » (contrôleur de gestion de la SICAP). Le fonctionnement des entreprises est assez bureaucratique aussi à la SN-HLM : "Pour chaque type de dépense, on vérifie en amont si la ligne budgétaire existe. En aval, on fait signer et on suit. On procède à des contrôles inopinés au niveau de la caisse et à la vérification des effectifs au niveau du travail. » qu'à la SICAP : "Pour le bon fonctionnement de l'entreprise, il a été mis en place un manuel des procédures qui représente, pour le contrôle de gestion, un instrument de pilotage de base. ». A la SICAP, Société d'Economie Mixte où les capitaux privés sont minoritaires mais présents, le contrôle de gestion est toutefois plus développé en hommes et en outils mis en œuvre qu'à la SN-HLM avec un reporting régulier et un suivi actif des écarts budgétaires. Dans les deux cas les calculs de coûts restent très réduits car les sociétés sont des donneurs d'ordre qui font appel à des entreprises du bâtiment extérieures pour construire leurs logements sur la base d'appels d'offre et de devis.

Dans la branche des détergents et produits d'hygiène, quatre entreprises se partagent le marché : la Savonnerie Africaine Fakhry (SAF), Alminco (d'après le nom du quartier où est implanté l'usine), la Société Sénégalaise de Savonnerie (3S) et Colgate-Palmolive-NSOA. La SAF appartient à une famille sénégalaise d'origine libanaise et est le leader sur le marché des savons. Le PDG a longtemps été le président de la Fédération Sénégalaise de Football et sa marque est très connue notamment parce qu'elle sponsorise beaucoup de matchs de football. La SAF ne dispose pas de comptabilité analytique ni de contrôle de gestion formalisé. L'équipe dirigeante est choisie dans le cercle familial ou dans la communauté libanaise du Sénégal. La supervision directe est effectuée par ces cadres qui ont une bonne formation en gestion. De son côté, Alminco est issue d'une évolution complexe. En 1929 une famille danoise crée au Sénégal la Nouvelle Savonnerie de l'Ouest Africain (NSOA). En 1993 l'entreprise est rachetée par Colgate-Palmolive qui revend ensuite en 2008 la production du savon «Le Marabout » à une équipe de privés sénégalais dont le fils de l'ancien directeur de la NSOA. En attendant la structuration du contrôle de gestion, c'est le chef du personnel, présent depuis 20 ans dans l'entreprise qui exerce la fonction. Le cabinet d'experts comptables qui suit l'entreprise joue un rôle déterminant: "On n'est pas encore arrivé à mettre en place un système budgétaire, ni un service autonome de contrôle de gestion. Tout est fait par le cabinet. Pour cela, on a en interne deux comptables qui sont en interface entre l'entreprise et le cabinet pour les informations et les documents de base. » (chef du personnel).

Orange-SONATEL est dominateur sur le marché des télécoms au Sénégal (leader sur la téléphonie mobile et en situation de quasi monopole sur la téléphonie fixe) et a des filiales dans toute la région (en Guinée-Bissau, au Mali, en République de Guinée). L'implication de la maison mère, France-Télécom est très importante et il s'agit d'un fleuron de l'économie sénégalaise. Le dispositif de pilotage de la performance est très complet (assez comparable à ce qui est mis en œuvre en France) et une comptabilité de gestion ABC a été installée en 2005. Dans le secteur de la téléphonie mobile, Tigo est une société anonyme créée en 1998 et appartenant au groupe luxembourgeois Millicom International Cellular. Elle est dotée d'une technologie avancée et ses parts de marché augmentent régulièrement. Son personnel est 
majoritairement composé de cadres formés à l'étranger. Le suivi budgétaire est très développé.

Dans le secteur agro-alimentaire, les deux entreprises étudiées ont comme activité principale la transformation du lait. Nestlé, présent au Sénégal depuis 1961, est la première entreprise alimentaire du pays. L'organisation interne est très structurée et le contrôle de gestion est très développé avec toute une batterie d'outils. Le contrôle de gestion est autonome par rapport à la direction financière : "L'autonomie du contrôle de gestion favorise son plein exercice. » (contrôleur de gestion Nestlé). De son côté, la SATREC, créée en 1992 par deux Sénégalais sans compétences particulières en gestion, utilise des technologies parmi les plus récentes de l'industrie laitière. Il n'y a pas de contrôleur de gestion, pas de budgets ni de tableaux de bord d'ensemble mais un pilotage direct par le directeur financier. Il y a un budget de trésorerie suivi régulièrement et des calculs de coûts complets (avec mise en place très récente de la méthode $\mathrm{ABC}$ se substituant à la méthode traditionnelle par les centres d'analyse) mais pas de coûts standards ni d'analyse d'écarts.

Dans le secteur bancaire nous avons pu étudier le contrôle de gestion de deux banques importantes appartenant toutes les deux au groupe bancaire marocain Attijari Wafa (dont l'actionnaire principal est la famille royale marocaine). La CBAO est possédée à $80 \%$ par Attijari Wafa. D'après le contrôleur de gestion rencontré : «La banque est le lieu d'utilisation d'une haute technologie de l'information et de la communication, d'où la nécessité de disposer à tous les endroits d'un personnel qualifié et d'un contrôle de gestion fortement instrumentalisé tant au plan du fonctionnement que de l'organisation. ». Pour ce qui relève des pratiques: «Il y a un progiciel de comptabilité bancaire qui permet de faire les comptabilités analytique, financière et budgétaire. La comptabilité analytique permet de calculer le minimum de coûts pour fixer les conditions de banque. Nous ignorons les méthodes poussées comme l'ABC. On fait souvent du benchmarking mais il y a plus de mimétisme que de calculs réels de coûts comparés. ». Le Crédit Agricole Sénégal, fililale jusqu'en 2009 du Crédit Agricole de France, vient d'être cédé en 2009 à Attijari Wafa mais la CBAO et le CAS n'ont pas fusionnés et gardent des statuts différents. Au CAS, il n'y a pas de service de contrôle de gestion en tant que tel et c'est le directeur finances et comptabilité que nous avons rencontré. Les pratiques de contrôle de gestion semblent assez réduites, peu structurées et très peu instrumentées.

Que peut-on en déduire?

Des entretiens réalisés (dont les éléments cités ci-dessus ne reflète qu'une partie des informations collectées), il ressort que les outils d'aide à la décision et les dispositifs d'orientation des comportements sont fortement développés chez Orange-SONATEL (grande entreprise, filiale de firme multinationale, télécom), chez Tigo (grande entreprise, filiale de firme multinationale, télécom), chez Nestlé Sénégal (grande entreprise, filiale de firme multinationale, agro-alimentaire) et chez CBAO (grande entreprise, filiale de firme multinationale, banque commerciale). On a affaire là, dans des univers très technologiques, à un contrôle de gestion structuré, managérial (assez semblable à ce qu'on observe dans les pays développés où la société mère des groupes est installée) et ayant certains aspects relevant du contrôle interactif au sens de Simons.

Les entretiens réalisés chez SN-HLM (entreprise moyenne, publique, aménagement immobilier) montrent un faible développement de l'instrumentation du contrôle, de fortes pressions exercées par l'environnement politique et l'importance des procédures 
administratives et juridiques qui caractérisent un contrôle financier et procédural très bureaucratique (Mintzberg, 1994). C'est également le cas pour SICAP (entreprise moyenne, parapublique, aménagement immobilier) avec un peu moins de rigidité et un peu plus d'outils de contrôle de gestion. On est là dans des systèmes de régulation par les procédures. Ceci est également le cas, de façon assez surprenante et avec les pressions politiques en moins, pour le CAS (grande entreprise, filiale de firme multinationale, banque commerciale).

Le contrôle de gestion formalisé est peu présent chez SATREC (entreprise moyenne, sénégalaise, agro-alimentaire), chez SAF (entreprise moyenne, sénégalaise, savonnerie) et chez Alminco (entreprise moyenne, sénégalaise, agro-alimentaire). On a ici affaire à un contrôle organisationnel fondé sur la supervision directe par les dirigeants et leurs proches, avec un système de valeurs partagées (si on reprend la typologie de Simons) et avec une instrumentation réduite.

Ces résultats recueillis sur un nombre réduit d'entreprises doivent maintenant être synthétisés dans un ensemble d'hypothèses généralisables et testables statistiquement à plus grande échelle sur un échantillon représentatif. Celles-ci, découlant donc de l'analyse des verbatims d'entretiens réalisés, ont trait aux trois styles de contrôle diagnostiqués sur la dizaine d'entreprises étudiées. Certaines hypothèses peuvent sembler évidentes, mais elles permettent de vérifier (ou non) si les facteurs de contingence classiques ou populaires dans les pays développés sont aussi une réalité dans le contexte de pays en développement à l'instar du Sénégal.

Les premières hypothèses visent à tester la différence d'instrumentation entre les filiales de firmes multinationales et les autres entreprises. Ces hypothèses sont :

- H1: Le contrôle de gestion est plus développé dans les filiales de firmes multinationales que dans les entreprises locales.

- H2 : Le contrôle de gestion est plus développé dans les grandes entreprises que dans les PME.

- H3 : Le contrôle de gestion est plus développé si les dirigeants ont une formation en gestion.

D'autres hypothèses visent à caractériser les modes de contrôle de gestion dans les PME familiales et dans les entreprises publiques ou parapubliques :

- H4 : A défaut d'un contrôle de gestion structuré, il existe des pratiques prudentielles que les petites et moyennes entreprises mettent en ouvre et qui rentrent dans le cadre d'un contrôle organisationnel.

- H5: Le contrôle de gestion des entreprises publiques se résume à l'élaboration et au suivi budgétaire.

D'autres hypothèses visent à tester l'influence de certains facteurs sur le développement de l'instrumentation du contrôle :

- H6 : Les démarches de réduction des coûts sont l'apanage des entreprises du secteur privé soumis à une forte concurrence.

- H7 : Les entreprises autochtones ont généralement recours aux consultants externes pour la mise en œuvre des techniques de contrôle de gestion. 


\section{Enquête quantitative confirmatoire}

Une étude statistique a été menée par questionnaires. Nous avons demandé à l'Agence Nationale de la Statistique et de la Démographie (ANSD) une base de données des entreprises déclarées de plus de 50 salariés construite à partir de son répertoire CUCI (Centre Unique de Collecte d'Informations). Le nombre d'entreprises dans ce fichier filtré se monte à 600 . Nous avons acheté un fichier tiré au hasard de 220 entreprises et nous avons administré le questionnaire. Comme le nombre de retour n'était pas suffisant, nous avons renouvelé l'opération avec un fichier complémentaire de 90 entreprises toujours tirées au hasard par secteurs à partir de la base de données. Le questionnaire a donc été ventilé auprès de 310 entreprises au total en deux vagues et 130 retours significatifs ont pu être analysés (cf. détail dans le tableau 2, ci-dessous).

Un pré-test du questionnaire a d'abord été effectué afin de permettre de réajuster les questions en cas de non compréhension ou de mauvaise formulation. Les questionnaires, avec leurs variables de contexte, causales et expliquées (cf. extraits en annexe 2) ont été administrés par dix enquêteurs (étudiants de masters de gestion formés et encadrés par un des auteurs). Une lettre était envoyée préalablement au directeur de l'entreprise pour lui faire part de la venue des enquêteurs et de l'objet de la recherche. Ensuite les étudiants sondeurs se rendaient dans l'entreprise pour poser les questions et remplir le questionnaire. Les interrogations étaient sous la forme de questions fermées par «oui » ou «non », de questions à choix multiples et d'échelles de Lickert. Les cibles de l'enquête ont été les contrôleurs de gestion ou les directeurs financiers dans les entreprises où la fonction existe et les dirigeants dans les entreprises où la fonction est absente. Beaucoup de difficultés pratiques (disponibilité réduite des sondés, réticences à répondre, problèmes de non connaissance réelle par les sondés de la nature de certains outils, etc.) ont dû être surmontées lors cette l'enquête. 
Tableau 2 : L'échantillon des 130 entreprises ayant répondu au questionnaire

\begin{tabular}{|c|c|c|c|c|c|c|}
\hline $\begin{array}{l}\text { Secteurs } \\
\text { d'activité }\end{array}$ & Métiers & $\begin{array}{c}\text { Filiales } \\
\text { de firmes } \\
\text { multinationales }\end{array}$ & $\begin{array}{c}\text { Entreprises } \\
\text { publiques } \\
\text { ou } \\
\text { parapubliques }\end{array}$ & $\begin{array}{c}\text { Entreprises } \\
\text { moyennes } \\
\text { privées } \\
\text { familiales } \\
\text { ou non }\end{array}$ & Envois & Retours \\
\hline Industrie & $\begin{array}{l}\text { Transformation agro- } \\
\text { alimentaire ; Production } \\
\text { de biens non } \\
\text { alimentaires ; Bâtiment et } \\
\text { Travaux Publics ; } \\
\text { Artisanat. }\end{array}$ & 20 & 17 & 23 & 60 & 30 \\
\hline Services & $\begin{array}{l}\text { Téléphonie ; Energie- } \\
\text { Electricité ; Hôtellerie- } \\
\text { Restauration ; Services } \\
\text { portuaires et } \\
\text { aéroportuaires ; } \\
\text { Entretien, réparation, } \\
\text { maintenance ; Logistique } \\
\text { et transports maritimes et } \\
\text { aériens ; Location de } \\
\text { voitures ; Etudes de } \\
\text { marché et projets ; } \\
\text { Etablissements de santé ; } \\
\text { Services bancaires et } \\
\text { assurance ; Couture. }\end{array}$ & 53 & 35 & 72 & 150 & 63 \\
\hline \multirow[t]{4}{*}{ Commerce } & $\begin{array}{l}\text { Grandes surfaces ; } \\
\text { Concessionnaires } \\
\text { automobiles ; Grande } \\
\text { distribution en produits } \\
\text { cosmétiques ; Grande } \\
\text { distribution en produits } \\
\text { électroménagers ; } \\
\text { Importateurs de denrées } \\
\text { alimentaires. }\end{array}$ & 40 & - & 50 & 90 & 37 \\
\hline & $\begin{array}{l}\text { Envois } \\
\end{array}$ & 113 & 52 & 145 & $\begin{array}{c}\text { Total : } \\
310\end{array}$ & \\
\hline & Retours & 26 & 20 & 84 & & $\begin{array}{c}\text { Total : } \\
\text { 130 }\end{array}$ \\
\hline & $\begin{array}{c}\text { Taux de retour: } \\
42 \% \text { sur l'ensemble }\end{array}$ & $23 \%$ & $38 \%$ & $58 \%$ & & \\
\hline
\end{tabular}

L'étude statistique a consisté dans un premier temps à voir si les facteurs de contingence décelés lors de l'enquête exploratoire se confirmaient sur la population de l'échantillon. Le logiciel «Spad» a permis de procéder à une caractérisation automatique qui constitue une forme d'analyse discriminante. Les données des tris croisés générés ont fait l'objet du test du Khi-deux avec des résultats concluants. Les résultats statistiques affichés par les tris croisés validés ont permis de faire des analyses typologiques avec segmentation des populations. En raison des limites et des biais bien connus des enquêtes par questionnaires, surtout sur des questions assez techniques telles que la nature et l'usage des outils de gestion dans les entreprises d'un pays peu développé économiquement, un usage modéré des méthodes statistiques a été effectué et nous en sommes restés aux techniques robustes et aux déductions assez simples. Nous avons également été très modestes et prudents dans la prise en compte des déclarations sur l'existence de certains outils car il nous semblait, après recoupements, que parfois les réponses surestimaient largement la réalité de terrain (par exemple dans 
l'usage déclaré de la méthode $\mathrm{ABC}$ ). Dans ce type d'enquête par questionnaires, le répondant peut en effet développer un biais systématique pour se valoriser et par souhait de paraitre moderne en déclarant avoir l'outil alors que ce n'est pas vrai. Le déclarant peut se tromper de bonne foi sur la nature de l'outil en lui attribuant une «étiquette » inadaptée. Il peut estimer que l'outil existe parce qu'il le connaît mais sans qu'il soit disponible dans l'entreprise. L'outil peut être disponible sans être utilisé. Il peut également être utilisé sans apporter satisfaction.

Les hypothèses formulées ont toutes été validées statistiquement par des tests du Khi-deux (cf. annexe 3).

\section{Discussion}

Il y a bien évidemment des différences dans les pratiques de contrôle de gestion selon le métier ou les caractéristiques de l'activité et cela est valable aussi bien dans les pays développés comme la France que dans les pays moins avancés comme le Sénégal. Ainsi par exemple, les comptabilités de gestion sont plus développées dans l'industrie où la chaîne de valeur gérée directement par l'entreprise a une épaisseur certaine que dans les activités d'intermédiation ou commerciales où il y a peu de complexité à gérer et de charges à optimiser entre les recours au marché en amont (achats des marchandises) et en aval (revente des marchandises). De même, on comprend bien que les calculs de coûts sont plus utiles dans les activités multiproduits que quand l'entreprise ne délivre qu'un seul produit. Il est logique, encore, que dans la distribution ou les services, les pratiques de benchmarking entre les différentes unités, points de vente ou agences relevant de l'entreprise en réseau soient fréquentes. On devine aussi facilement que dans les activités stables, en phase de maturité de production ou pour les Domaines d'Activité Stratégiques (DAS) de type « vaches à lait » les budgets et le suivi de trésorerie sont centraux alors que pour les activités innovantes, les produits en phase de lancement ou pour les DAS de type « vedettes » ou «dilemmes » l'usage des tableaux de bords multidimensionnels sera plus adapté. Tout ceci n'est pas spécifique aux pays du Sud.

Nous avons donc plutôt centré notre réflexion sur les éléments caractéristiques des pratiques de contrôle de gestion dans un pays comme le Sénégal, par rapport à la nature de son tissu économique. Il ressort des résultats de l'enquête que dans la mise en œuvre du contrôle de gestion, l'élément majeur, différenciant les pratiques de contrôle de gestion au Sénégal, reste la distinction entre les filiales de firmes multinationales, les entreprises publiques et parapubliques et les entreprises privées sénégalaises familiales ou non.

Les filiales de firmes multinationales (environ $15 \%$ de l'échantillon exploitable) ont les caractéristiques suivantes :

- un développement du contrôle de gestion comparable à ce qui est constaté dans les maisons mères des pays développés ;

- $\quad$ un niveau de formation des employés élevé ;

- une forte structuration de la fonction contrôle de gestion (existence du poste dans 3/4 des entreprises) ;

- un recours aux cadres internes pour la mise en œuvre des outils de contrôle de gestion ( $2 / 3$ des cas) ;

- une diffusion plutôt rapide des techniques de contrôle de gestion ; 
- une forte expérience des dirigeants dans l'usage des techniques de contrôle de gestion ;

- une instrumentation de gestion très développée au niveau opérationnel (un peu plus de $75 \%$ des cas) ;

- des logiciels de contrôle de gestion très utilisés (80\% des cas) ;

- des calculs des coûts très développés (dans $80 \%$ des entreprises).

Les entreprises publiques et parapubliques (environ 20\% de l'échantillon exploitable) présentent les traits suivants :

- un contrôle de gestion peu développé et un fonctionnement bureaucratique avec prise en compte réduite de la contrainte économique et des dimensions efficience et efficacité ;

- un niveau de formation des employés plutôt élevé mais pas forcément dans le domaine de la gestion ni dans la technique ;

- une assez forte structuration de la fonction contrôle de gestion (existence du poste dans $2 / 3$ des cas) ;

- une diffusion lente des techniques de contrôle de gestion ;

- un recours modéré aux consultants externes pour la mise en place des outils de contrôle de gestion ( $40 \%$ des cas constatés) ;

- une instrumentation de gestion développée au niveau opérationnel ( $2 / 3$ des cas) ;

- peu de logiciels de contrôle de gestion (seulement dans $25 \%$ des cas) ;

- un calcul des coûts très peu développé (dans $1 / 4$ des cas seulement);

- une régulation par l'Etat du fonctionnement des entreprises par la mise en place de manuels de procédures administratives et financières (environ $50 \%$ des cas);

- un contrôle de gestion plutôt fondé sur le respect des règles dans les entreprises purement publiques.

Les entreprises privées sénégalaises familiales ou non (environ $65 \%$ de l'échantillon exploitable) ont comme caractéristiques :

- un niveau de formation plutôt faible pour ce qui concerne les entreprises purement familiales mais présence d'employés de niveau supérieur ou égal à « $\mathrm{Bac}+2$ » aux postes de responsabilité dans de nombreuses PME non familiales ;

- une faible structuration de la fonction contrôle de gestion (poste dans seulement $20 \%$ de ces entreprises);

- un recours fréquent aux consultants externes fort pour la mise en œuvre des outils de contrôle de gestion (dans 3/4 des cas) ;

- une diffusion lente des techniques de contrôle de gestion ;

- une supervision directe prédominante au niveau opérationnel ;

- peu ou pas de logiciels de contrôle de gestion (usage dans moins de $15 \%$ des entreprises) ;

- une instrumentation de gestion faible au niveau opérationnel (moins de $10 \%$ des cas) ;

- un calcul des coûts assez développé (dans environ la moitié des entreprises).

Plusieurs voies d'améliorations pour les PME ressortent de l'enquête. Des calculs de coûts partiels et de marges sont souvent plus utiles dans les PME que des modélisations d'ensemble et le calcul de coûts complets et résultats par produits. Des systèmes budgétaires sophistiqués n'ont pas de sens si l'environnement est très turbulent. L'élaboration de tableaux de bord n'est utile que si les leviers d'action existent permettant d'agir à partir de ces informations. Par ailleurs on voit bien l'intérêt pour les PME de se doter de dispositifs simples pour établir des devis et répondre à des appels d'offre, l'intérêt des business plans et calculs de seuils de 
rentabilité, la mise en œuvre de démarches de réduction des coûts, etc. Dans tout ce qui concerne les outils d'aide à la décision, les voies d'amélioration pour les PME autochtones sont donc faciles à identifier. Des comparaisons sont également possibles avec ce qui a été mis en évidence par Ngongang au Cameroun (2005) et au Tchad (2007) relativement à l'ensemble des informations comptables utilisées par les PME. Par contre pour ce qui concerne les outils assurant la convergence des comportements, la dimension humaine des organisations est essentielle et, là, ce sont les facteurs sociaux et culturels qui jouent un rôle crucial. Des observations in situ, des études de terrain et des méthodes qualitatives plus approfondies seront alors nécessaires pour faire des préconisations motivées.

Il paraît également, à l'issue des entretiens et du dépouillement du questionnaire, qu'il faudrait accroître la qualité de la formation initiale des jeunes managers nouvellement recrutés. La responsabilité du système éducatif est ici essentielle. Les formations au contrôle de gestion doivent être développées et proposer aux futurs cadres des méthodologies et des outils adaptés permettant de répondre aux besoins divers des entreprises : donner des éléments structurants en contrôle de gestion pour les managers de terrain des PME autochtones et former les spécialistes du contrôle de gestion pour les grandes entreprises qui sont en nombre réduit et dépendent souvent de groupes internationaux. La socialisation interne, dans l'entreprise, des managers intermédiaires et de terrain autour des méthodes modernes de contrôle de gestion relève ensuite de la responsabilité des entreprises et nécessite un dosage fin et évolutif entre l'existant (cultures nationales des employés et faible développement du tissu économique) et le souhaitable (référents universaux en matière de responsabilité individuelle et impératif de modernisation économique et sociale dans la mondialisation).

\section{Conclusion}

Dans les filiales de firmes multinationales il y a un contrôle managérial avec une instrumentation développée au niveau opérationnel et une acculturation des managers dans une démarche de formation et de développement des capacités des acteurs. Par certains aspects, il semble se rapprocher dans la plupart de ces entreprises d'un contrôle « interactif » au sens de Simmons (en tous cas par comparaison aux pratiques des autres entreprises dans le contexte sénégalais). Dans les entreprises publiques ou parapubliques, le contrôle est procédural, financier avec un pilotage par les règles, sous l'autorité directe ou indirecte de l'Etat, avec des manuels de procédures et un faible développement de l'instrumentation de gestion. Les calculs des coûts sont peu développés, les budgets sont pesants et sont plus une contrainte (ce qui peut se justifier dans le secteur relevant directement du service public financé par le contribuable) qu'un système de management permettant la mise sous tension de l'organisation et assurant la convergence des comportements. On relève là des dimensions de « respect des règles » (surtout) et de «contrôle diagnostique » (un peu) si on reprend la typologie des leviers du contrôle de Simons. Dans les PME locales, le pilotage de la performance se fait essentiellement par la supervision directe du chef d'entreprise et des membres de sa famille au sens large qui travaillent dans l'entreprise. La culture nationale, les traditions ethniques et les relations interpersonnelles jouent un rôle déterminant. La planification stratégique est la plupart du temps inexistante et l'instrumentation de gestion peu développée. On a un contrôle de gestion embryonnaire. La diffusion des techniques et des outils du contrôle de gestion dans les entreprises locales purement sénégalaises est donc très lente. Quand les problèmes de gestion deviennent trop importants ou quand l'équipe de direction de la PME (propriétaires dirigeants et famille élargie ou associés proches) souhaite mettre en place une démarche de rationalisation de la gestion souvent en s'appuyant sur des outils informatiques, le recours aux consultants externes semble la règle. 
La contribution scientifique principale de cette étude réside dans la connaissance des pratiques en contrôle de gestion dans les pays en développement, ici le Sénégal. L'apport pour les professionnels est dans la mise en évidence des voies d'amélioration de l'instrumentation au Sénégal surtout dans les PME autochtones. Les limites de cette recherche sont son côté essentiellement descriptif et le fait que nous avons délibérément privilégié la dimension «système d'information » (au sens le plus large du terme) du contrôle de gestion, dans une recherche symétrique en quelque sorte de celle de Kaboré (2010) consacrée à la dimension « système d'animation » du contrôle de gestion au Burkina-Fasso. Les perspectives futures de recherche sont à chercher d'une part dans des études comparatives en Afrique sub-saharienne (pour élargir le constat à l'échelle du continent) et d'autre part dans le développement de recherches «action » permettant d'observer et d'améliorer le mouvement concret des acteurs s'appropriant les outils et les mettant en œuvre efficacement (dans une approche dynamique du processus de modernisation de la gestion interne des entreprises dépassant l'opposition figée entre des outils qui seraient de par leur origine «occidentaux» et des cultures qui seraient immobiles dans leur « africanité »). 


\section{Bibliographie}

Abrahamson, E. (1991). Managerial Fads and Fashions: The Diffusion and Rejection of Innovations. Academy of Management Review 16 (3): 586-612.

Alcouffe, S., Berland, N., Levant, Y. (2003). Les facteurs de diffusion des innovations managériales en comptabilité et contrôle de gestion : une étude comparative. Comptabilité - Contrôle - Audit. numéro spécial, mai: 7-26.

Anthony, R. (1965). Planning and Control Systems: a Framework for Analysis. Division of Research, Graduate School of Business, Harvard University, Boston.

Anthony, R., Govindarajan, V. (1998). Management Control Systems. Mc Graw Hill.

Bellot, J.-M. (1988). Petites et moyennes entreprises au Sénégal: quelques cas de success stories. Grenoble, IREP Développement, Cahier 26, mars.

Benabdeljlil, N. (2007). Les modes de management des entreprises au Maroc : entre contingences culturelles et économiques. Revue Internationale PME 20 (2): 89-122.

Berland, N. (2002). Le contrôle budgétaire. Collection Repères. Editions La Découverte.

Boungou Bazika, J.-C. (2005). Essai de définition et de fonctionnalité de l'entreprise familiale dans une perspective africaine. Revue Internationale PME 18 (3-4): 11-30.

Bouquin, H. (2008). Le contrôle de gestion. $8^{\text {ème }}$ édition. Collection Gestion. Paris. PUF.

Bourguignon, A. (2009). Performance et contrôle de gestion. In Collasse Eds Encyclopédie de Comptabilité, Contrôle de Gestion et Audit $2^{\text {ème }}$ édition. Economica: 1121-1132.

Busco, C., Giovannoni, E., Riccaboni, A. (2007). Globalisation and International Convergence of Management Accounting. In Hopper, Northcott et Scapens Eds: Issues in Management Accounting. London. Prentice Hall: 65-92.

Choffel, D., Meyssonnier, F. (2005). Dix ans de débats autour du Balanced Scorecard. ComptabilitéContrôle-Audit 11 (2): 61-81.

Donada, C., Nogatchewsky, G. (2008). Partenariat, vassalité, marché et seigneurie : 4 configurations de contrôle client-fournisseur. Comptabilité-Contrôle-Audit. 14 (1): 145-168.

Efferin, S., Hopper, T. (2007). Management Control, Culture and Ethnicity in a Chinese Indonesian Company. Accounting, Organizations and Society 32: 223-262.

Ferreira, A., Otley, D. (2009). The Design and Use of Performance Management Systems: An Extended Framework for Analysis. Management Accounting Research 20 (4): 263-282.

Gervais, M., Kaboré, S. (2007). Quels systèmes d'animation pour l'entreprise burkinabé ? Management International 11 (3): 43-73.

Gosselin, M., Mévellec, P. (2003). Plaidoyer pour la prise en compte des paramètres de conception dans la recherche sur les innovations en comptabilité de gestion. Comptabilité-Contrôle-Audit, numéro spécial, juin: 45-57. 
Goullet, C., Meyssonnier, F. (2009). Le contrôle des réseaux de franchise. Comptabilité-ContrôleAudit 17 (1): 99-122.

Hernandez, E. (1993). La gestion particulière des entreprises du secteur informel : le cas africain. Direction et Gestion n ${ }^{\circ} 41-142$ : 49-64.

Hernandez, E. (1999). Aspects financiers et comptables de l'entreprise informelle africaine. Gestion 24 (1): 68-75.

Hofstede, G. (1980). Culture's Consequences: International Différences in Work-Related values, Beverly Hills, Sage.

Kamdem, E., Ongodo, M. (2004). Faits et méfaits de l'ethnicité dans les pratiques managériales au Cameroun. Actes du colloque du réseau de recherche en sciences de gestion de l'AUF, Beyrouth, 2829 octobre.

d'Iribarne, P. (1989). La logique de l'honneur. Paris, Seuil.

d'Iribarne, P. (2007). Islam et management. Le rôle d'un univers de sens. Revue Française de Gestion 3 (171): 141-156.

Joannides, V. (2011). Apport de l'ethnicité à la culture en sciences de gestion. Finance-ContrôleStratégie 14 (1): 33-68.

Kaboré, S. (2010). Systèmes d'animation du contrôle de gestion dans les entreprises burkinabé : étude transculturelle. Thèse soutenue le 16 décembre sous la direction du professeur Gervais à l'Université Rennes 1.365 pages.

Kaplan, R., Norton, D. (2001). Comment utiliser le tableau de bord prospectif. Paris. Les Editions d'Organisation. Paris.

Lambert, C., Sponem, S. (2009). La fonction contrôle de gestion : proposition d'une typologie. Comptabilité-Contrôle-Audit 15 (2): 133-144.

de La Villarmois, O., Levant, Y. (2010). La mise en place et l'utilisation de la méthode UVA : une comparaison de deux enquêtes réalisées en 2001 et 2009. In Gervais Eds La comptabilité de gestion par les méthodes d'équivalence. Eyrolles. Paris.

Löning, H. (1995). A la recherche d'une culture européenne en comptabilité et contrôle de gestion. Comptabilité - Contrôle - Audit 2 (1): 81-97

Mévellec, P. (2005). Les systèmes de coûts. Dunod.

Meyssonnier, F. (2001). Le target costing : un état de l'art. Finance-Contrôle-Stratégie 4 (4): 113-138.

Meyssonnier. F. (2003). L'approche des coûts complets par les équivalents de production, voie d'avenir ou impasse ? Comptabilité-Contrôle-Audit 9 (1): 111-124.

Meyssonnier, F., Zawadzki, C. (2008). L'introduction du contrôle de gestion en PME : étude d'un cas de structuration tardive de la gestion d'une entreprise familiale en forte croissance. Revue internationale PME 21 (1): 69-92.

Meyssonnier, F., Rasolofo-Distler, F. (2008). Le contrôle de gestion entre responsabilité globale et performance économique : le cas d'une entreprise sociale pour l'habitat. Comptabilité-Contrôle-Audit 14 (2): 107-124. 
Meyssonnier, F. (2011). Le contrôle de gestion des services : spécificités, outils, enjeux. Actes du congrès de l'AFC de Montpellier. 24 pages.

Mintzberg, H. (1994). Structure et dynamique des organisations. $9^{\text {ème }}$ édition, Les Editions d'Organisation.

Moalla, H. (2007). Les mécanismes de diffusion, d'adoption et de rejet de la méthode ABC dans l'environnement tunisien. Actes du $28^{\text {ème }}$ congrès de l'AFC, Poitiers, mai.

Mouritsen, J. (1995). Management Accounting in Global Firms. In Ashton, Hopper et Scapens Issues in Management Accounting. London, Prentice Hall: 299-320.

Mzid Ben Amar, I., Mezghani, L. (2010). L'impact de la structure de la famille sur le développement des petites entreprises familiales en hypogroupe. Revue Internationale PME 23 (1): 125-154.

Ngongang, D. (2005). Pratiques comptables, système d'information et performance des PME camerounaises. La Revue des Sciences de Gestion, Direction et Gestion 216: 59-70.

Ngongang, D. (2007). Analyse des facteurs déterminants du système comptable et des pratiques comptables des PME tchadiennes. La Revue des Sciences de Gestion, Direction et Gestion 224-225: 49-57.

Ngongang, D. (2010). Analyse de la pratique des coûts dans les PMI camerounaises. Revue libanaise de gestion et d'économie, 5: revue électronique, 22 pages.

Nobre, T. (2001). Méthodes et outils du contrôle de gestion dans les PME. Finance-Contrôle-Stratégie 4 (2): 119-148.

Quattrone, P., Hopper, T. (2005). A «Time-Space Odyssey » : Management Control Systems in Two Multinational Organisations. Accounting, Organizations and Society 30: 735-764.

Rogers, E. (1995). Diffusion of innovations. Free Press, $4^{\text {ème }}$ édition, New-York.

Simons, R. (1995). Levers of Control. Harvard University Press. Boston.

Wade, B. (2003). Contrôle de gestion et réforme hospitalière : cas du Sénégal. Revue africaine de gestion 1, avril. 22 pages.

Wickramasinghe, D., Hopper, T., Rathnasiri, C. (2004). Japanese Cost Management Meets Sri Lankan Politics: Disappearance and Reappearance of Bureaucratic Management Controls in a Privatised Utility. Accounting, Auditing \& Accountability Journal 17 (1): 85-120.

Yousfi, H. (2007). Gérer en Jordanie. Une coexistence problématique entre système hiérarchique et idéal religieux. Revue Française de Gestion 3 (171): 157-173. 
Annexe 1 : Guide d'entretien pour la phase exploratoire

\section{CARACTERISTIQUES DE L'ENTREPRISE}

- Quel est votre secteur d'activité ?

- En quoi consiste votre activité ?

- Quel est le statut juridique actuel de votre entreprise ?

- Quelle en est la structure ou l'origine du capital?

- Quel est le nombre de vos salariés?

- Quel est le niveau de votre technologie ?

- Par quels moyens faites-vous la concurrence?

- Y a-t-il des facteurs spécifiques qui complexifient la gestion de votre entreprise ?

\section{OUTILS DU CONTROLE DE GESTION MIS EN OEUVRE}

- Y a-t-il une comptabilité de gestion? si oui avec des coûts complets? (combien de centres d'analyse? quelle fréquence? quel usage ?), des coûts variables ? des coûts spécifiques ? des coûts spécifiques avec imputation rationnelle des charges indirectes? des coûts complets $A B C$ ? autre chose?

- Y a-t-il d'autres calculs des coûts ? si oui comment? à quelle occasion?

- Fait-on des devis ? si oui comment : au forfait, «à la louche » ou sur la base de calcul précis ?

- A-t-on des budgets? des tableaux de bord opérationnels? des tableaux de bord stratégiques (balanced scorecard) ?

- A-t-on des coûts prévisionnels ? des comparaisons ? des analyses d'écarts ?

- L'entreprise développe-t-elle un système de Gestion de la Production Assistée par Ordinateur (GPAO) ? un système d'administration des ventes?

- Y $\quad$ a-t-il un contrôle interne ? si oui comment et par qui est-il animé ?

\section{DEMARCHES, VOIES DE SOCIALISATION ET DE COMMUNICATION DU CONTROLE DE GESTION}

- Comment est organisé le contrôle de gestion?

- Y a-t-il un contrôleur de gestion ? qui fait quoi ?

- Quels sont les différents centres de responsabilité de l'entreprise ?

- Comment la délégation de pouvoir est-elle assurée?

- Y a-t-il des outils informatiques ? lesquels?

- Comment fait-on concrètement pour établir les comptes, les coûts et les indicateurs ?

- Comment élabore-t-on les budgets ?

- Quelles sont les procédures employées pour situer les responsabilités quant aux écarts constatés?

- Quels sont les moyens de supervision, d'incitation ou de coercition, de communication mis en place?

- Quels sont les facteurs qui vous ont conduit à adopter vos techniques et outils de gestion ?

- Qu'est-ce qui peut vous pousser à abandonner une technique ou un outil de gestion?

\section{FORCES ET FAIBLESSES DES RESSOURCES HUMAINES}

- Quels sont les niveaux de qualification de vos employés?

- Comment motivez-vous votre personnel?

- Sur quels critères faites-vous vos recrutements?

\section{CONTROLE DE GESTION ET PERFORMANCE FINANCIERE}

- Quels dispositifs et instruments utilise-t-on pour la création de la valeur? des centres de profit et EVA ? des PCI (et sur quelles bases?), des coûts complets par des centres d'analyses ? choix d'investissement (VAN, TRI ou multicritères)? des démarches TQM, JAT, ABC - ABM ? autre chose?

- A-t-on des techniques de réduction des coûts ? si oui l'Analyse de la Valeur ? le Kaizen Costing ? le Target Costing ? le Budget Base Zéro ? les Cost Killers ? Six Sigma?

- Fait-on du benchmarking? du reengineering? de la sous-traitance ? autre chose dans ce sens ? 


\section{NATIONALITE, TAILLE ET STATUT JURIDIQUE DE L'ENTREPRISE}

Etes-vous une entreprise :

Sénégalaise ? Etrangère non filiale? Etrangère filiale ?

Si vous êtes une entreprise étrangère, quelle est votre nationalité ?

Quel est l'effectif du personnel?

Etes-vous une entreprise :

Familiale purement sénégalaise? Publique ou parapublique?

Filiale de firme multinationale? Autre ? (à préciser)

A quel secteur d'activité appartenez-vous?

Services? Industrie? Commerce?

\section{CULTURE, FORMATION DES CADRES ET EXISTENCE DE CONTROLEURS DE GESTION}

Quel est le niveau de formation prédominant de vos cadres aux postes de responsabilité ?

Elevé $(\mathrm{Bac}+5$ et plus) ? Moins Elevé $(\mathrm{Bac}+2$ à +4$)$ ? Faible (inférieur à Bac +2$)$ ?

Avez-vous des contrôleurs de gestion?

\section{FACTEURS DE DIFFUSION DES TECHNIQUES DE CONTROLE DE GESTION}

Etes-vous dans un ou des cercles de partenariat ou dans un groupement d'entreprises permettant de poursuivre certains buts communs?

$\mathrm{Ce}(\mathrm{s})$ cercle ou ce(s) groupement vous aide-t-il à organiser, à mettre en place ou à développer certaines techniques de gestion?

Dans votre entreprise, comment les techniques de contrôle de gestion adoptées sont-elles mises en place ?

Progressivement? Immédiatement?

Avec l'aide de qui les techniques sont-elles installées dans votre entreprise ?

Les consultants externes seuls? Les cadres de l'entreprise en interne seuls?

Avec les consultants externes et les cadres en interne? Autres ? (à préciser)

\section{INSTRUMENTS DE MESURE, D'ANALYSE ET DE PILOTAGE DE LA PERFORMANCE}

Avez-vous des pratiques de calculs de coûts?

Pratiquez-vous la méthode du coût variable?

Pratiquez-vous la méthode du coût spécifique?

Pratiquez-vous la méthode du coût complet?

Pratiquez-vous la méthode du coût complet avec imputation rationnelle des charges fixes?

Pratiquez-vous la méthode $A B C$ ?

Pratiquez-vous une autre méthode ? (à préciser)

Avez-vous un dispositif de calculs d'écarts entre prévisions et réalisations?

Ya-t-il une gestion prévisionnelle ?

Avez-vous un service de comptabilité analytique ou une cellule de calcul des coûts ?

Le calcul des coûts est-il développé dans votre entreprise? 


\section{MECANISMES ET INSTRUMENTS DE DEPLOIEMENT ET DE PILOTAGE DE LA STRATEGIE}

Elaborez-vous des budgets?

Construisez-vous des tableaux de bord opérationnels?

Avez-vous un tableau de bord stratégique?

Avez-vous un contrôle interne?

Y a-t-il des logiciels conçus pour le contrôle de gestion dans votre entreprise ?

Faites-vous du reporting?

Vos responsables opérationnels élaborent-ils des rapports d'activité?

\section{ORGANISATION ET ROLE DU CONTROLE DE GESTION}

Quelle est la structure interne qui abrite le contrôle de gestion dans votre entreprise ?

Dans votre entreprise, quelles sont les tâches du contrôleur de gestion?

Si contrôle de gestion ne s'avère pas nécessaire pour votre entreprise, les tâches opérationnelles sont alors coordonnées :

Sous forme de supervision directe par le dirigeant principal

Par une standardisation des comportements à l'aide de règles de procédure

Autres (à préciser)

Existe-t-il un manuel de procédures dans votre entreprise? 


\section{Annexe 3 : Tests statistiques des hypothèses (extraits)}

Les conditions de réalisation de ce test sont réunies : l'effectif global dépasse 100 et les effectifs observés dans chacune des classes sont au moins égaux à 5. Un traitement automatique des données a été effectué via le logiciel «Spad». Nous présentons ici quelques extraits des principaux tris croisés possibles avec les variables du questionnaire issus de la caractérisation automatique. Les deux premières hypothèses formulées dans la phase qualitative $(\mathrm{H} 1$ et $\mathrm{H} 2)$ peuvent être corroborées par le tri ci-après.

\begin{tabular}{|c|c|c|c|c|c|c|c|}
\hline $\begin{array}{l}\text { Structure de la propriété } \\
\text { Utilisation de logiciels conçu }\end{array}$ & s pour le & ôle gestion & & & & & \\
\hline $\begin{array}{l}\text { Effectifs } \\
\text { \% ligne } \\
\end{array}$ & & Oui & & Non & & ENSEMBLE & \\
\hline Entreprises familiales sénégalaises & $9,5 \%$ & $16,2 \%$ & $90,5 \%$ & $61,3 \%$ & $100,0 \%$ & 63 & $48,5 \%$ \\
\hline Filiales de Firmes Multinationales & $80,8 \%$ & $56,8 \%$ & $19,2 \%$ & $5,4 \%$ & $100,0 \%$ & 26 & $20,0 \%$ \\
\hline Entreprises publiques et parapubliques & $25,0 \%$ & $13,5 \%$ & $75,0 \%$ & $16,1 \%$ & $100,0 \%$ & 20 & $15,4 \%$ \\
\hline Entreprises sénégalaises non familiales & $23,8 \%$ & $13,5 \%$ & $76,2 \%$ & $17,2 \%$ & $100,0 \%$ & 21 & $16,2 \%$ \\
\hline ENSEMBLE & $28.5 \%$ & $100,0 \%$ & $71.5 \%$ & $100,0 \%$ & $100.0 \%$ & 130 & $100,0 \%$ \\
\hline
\end{tabular}

Il ressort de ce tri que $80 \%$ de filiales de firmes multinationales (constituant de par leur taille les plus grandes entreprises) ont des logiciels conçus pour le contrôle de gestion, tandis que $90 \%$ d'entreprises familiales sénégalaises n'en ont pas.

En ce qui concerne l'hypothèse 3 , l'instrumentation de la gestion fait appel à de bons niveaux de qualifications de la part des employés. Le tri ci-après le prouve.

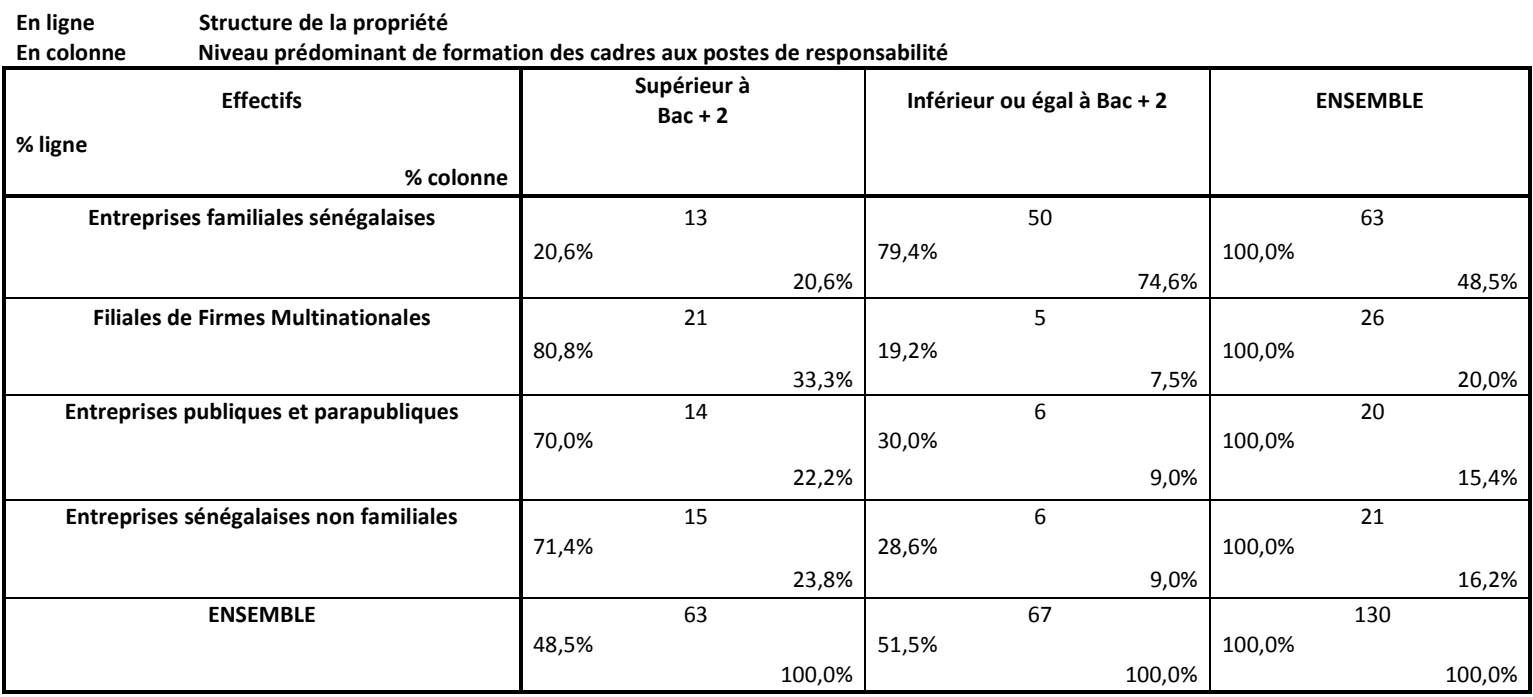

KHI2 $=38.55 / 3$ DEGRES DE LIBERTE / 0 EFFECTIFS THEORIQUES INFERIEURS A 5

PROBA $($ KHI2 $>38.55)=0.000 / \mathrm{V} . \mathrm{TEST}=5.48$ 
Dans seulement $20 \%$ des entreprises familiales sénégalaises, le niveau de formation prédominant des employés cadres aux postes de responsabilité est supérieur à «Bac +2 » contre en moyenne $74 \%$ pour les autres formes d'entreprises. Ici, il y a lieu de préciser que la formation des employés dont il s'agit est celle relative aux différents métiers exercés dans les entreprises. Ces métiers relèvent du domaine technique et/ou du domaine managérial.

Par rapport à l'hypothèse 4, les entreprises autochtones, d'après l'étude exploratoire qualitative, n'ont pas pour l'essentiel un contrôle de gestion structuré. Mais pour pallier cette insuffisance, elles mettent en place des manuels de procédures qui constituent des référentiels pour l'effectivité d'un contrôle organisationnel minimal. A travers le tri ci-après on voit que les catégories d'entreprises dans lesquelles le contrôle de gestion et le calcul des coûts ne sont pas développés disposent en général de manuels de procédures.

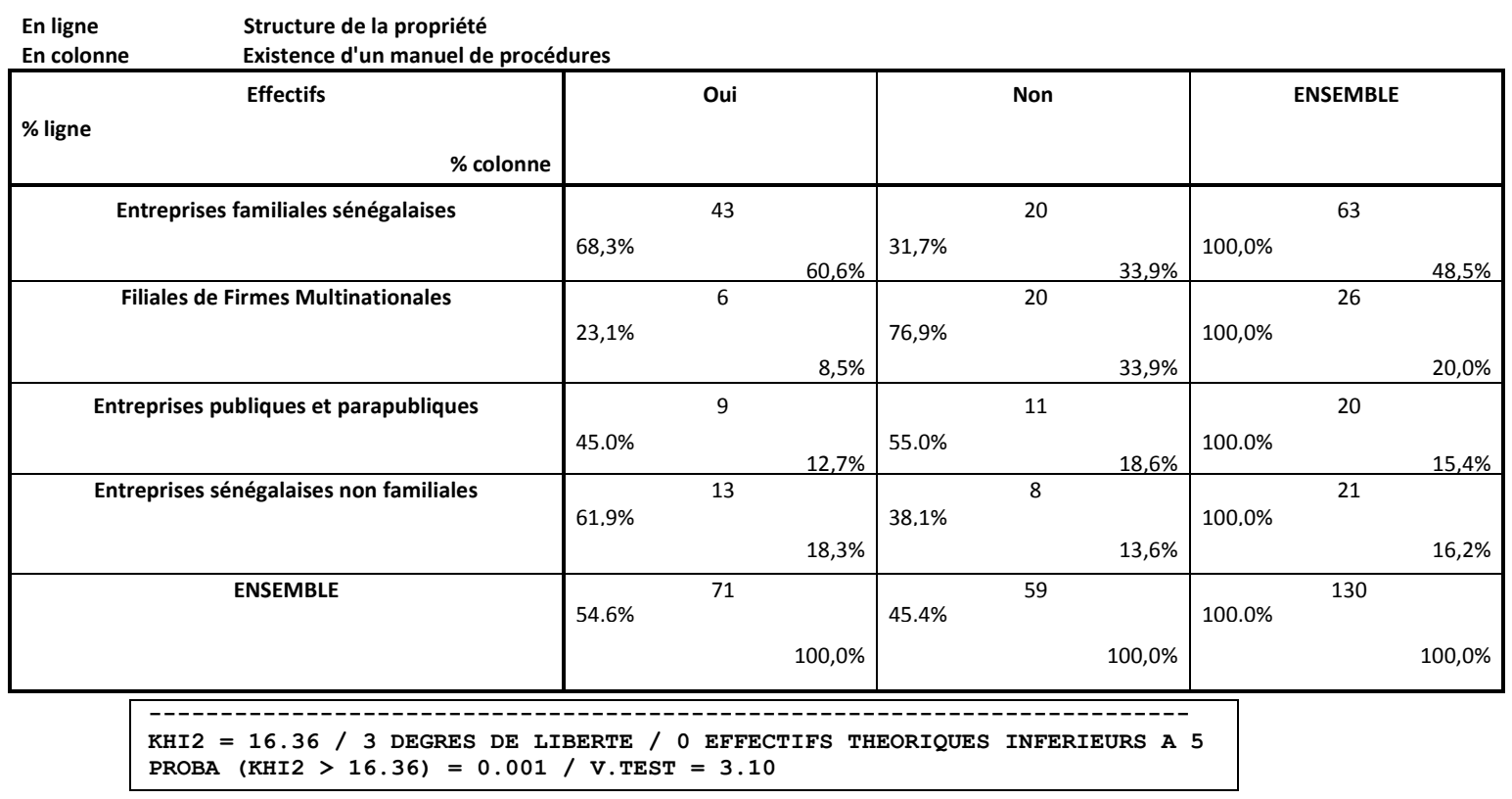


L'hypothèse 5 a fait l'objet de deux tris croisés ci-dessous présentés. D'abord les entreprises publiques et parapubliques pratiquent la gestion budgétaire comme les autres entreprises, ce qui apparaît dans le premier tri ci-dessous. Le tri suivant celui-ci montre que pour l'essentiel les entreprises publiques ne calculent pas les coûts.

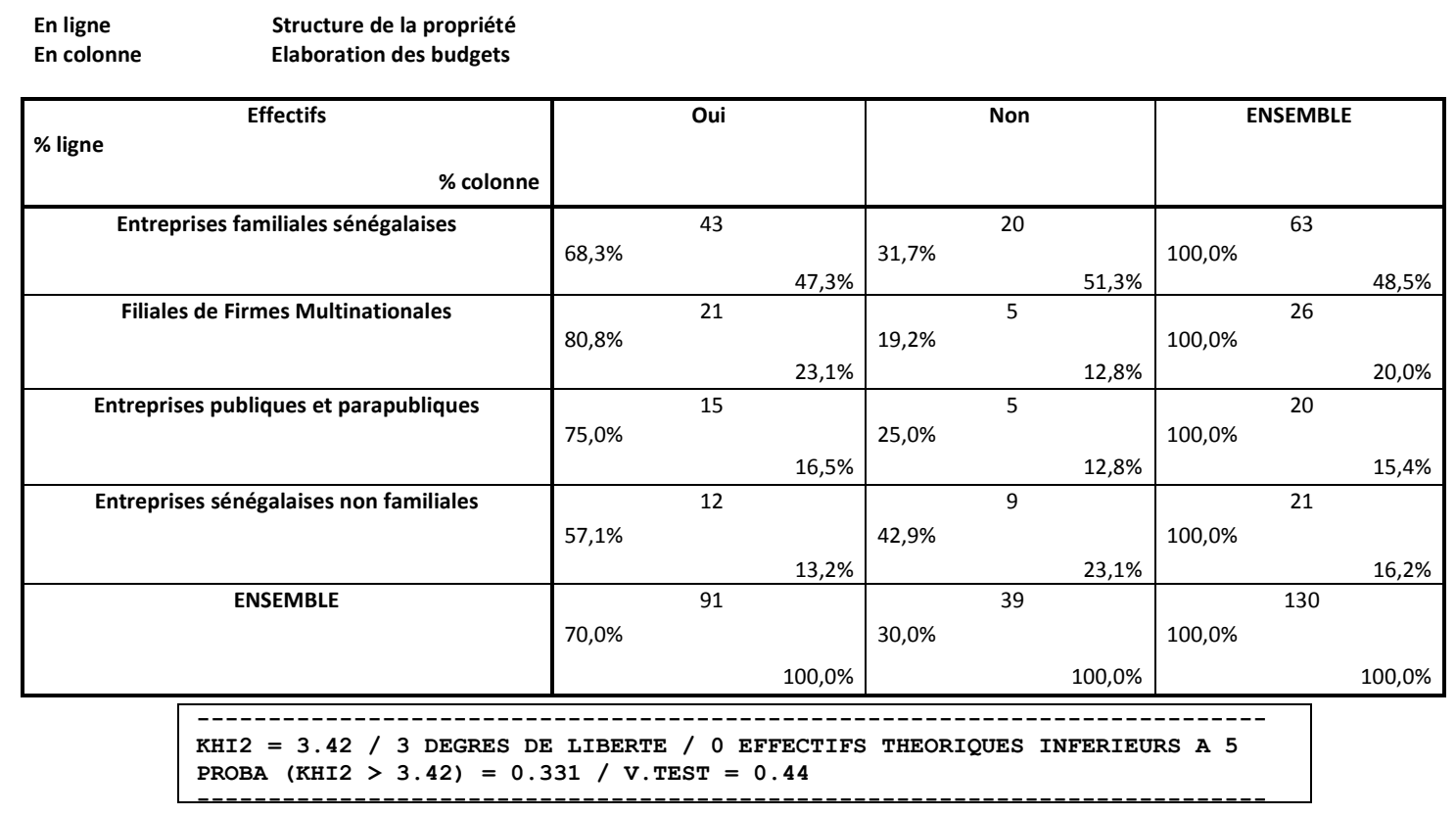

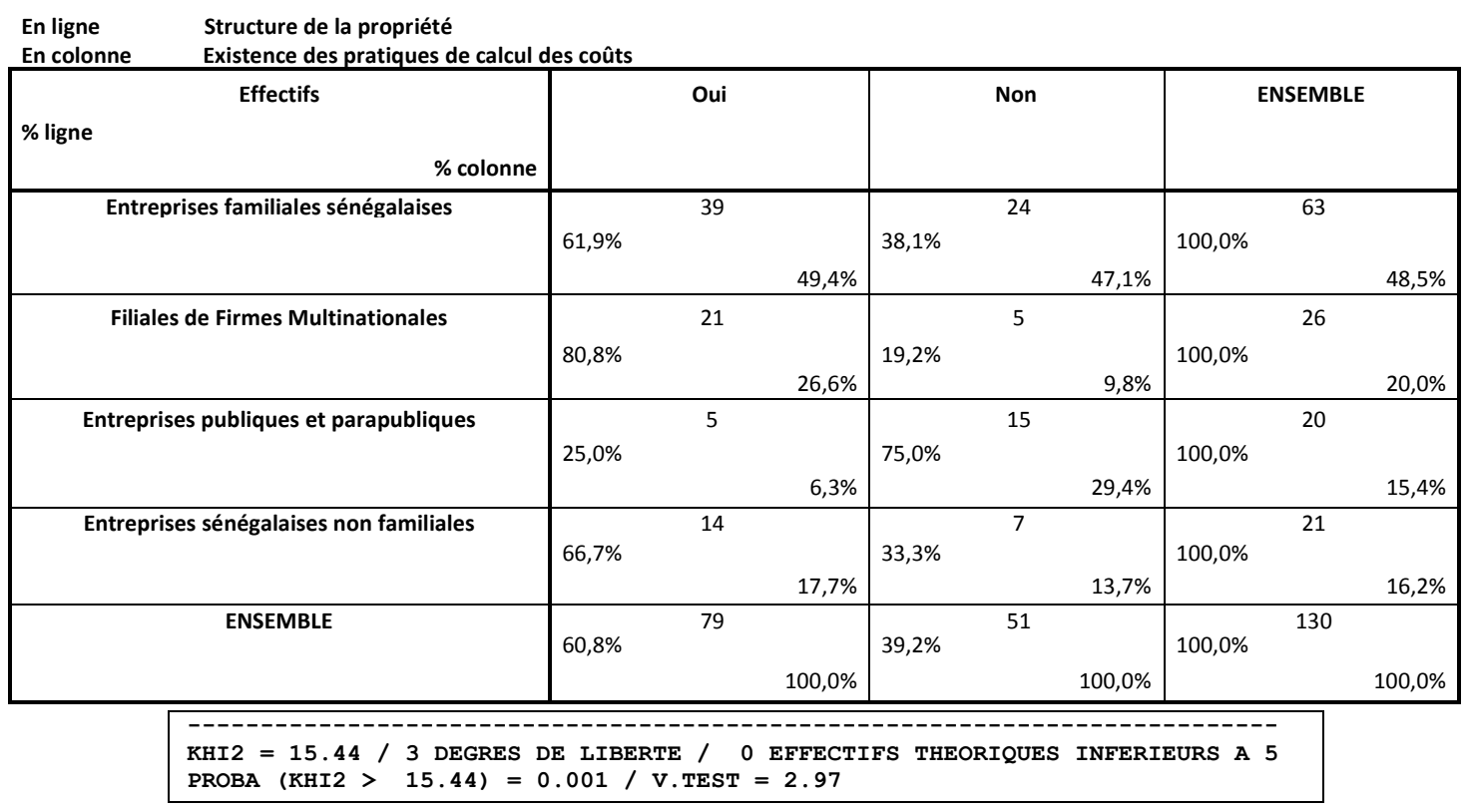

Concernant l'hypothèse 7 , le tableau ci-dessous montre une forte implication des cadres internes dans la mise en place des techniques de contrôle de gestion chez les filiales de firmes multinationales et les entreprises publiques et parapubliques, alors que cela nécessite, le plus souvent chez les entreprises privées sénégalaises familiales ou non familiales, une expertise externe qui justifie l'intervention des consultants. Autrement dit, il y a une absence assez notoire de cadres de conception dans les PME privées autochtones, ce qui au plan de pilotage de la performance n'est pas favorable à l'initiation des démarches de changement. 


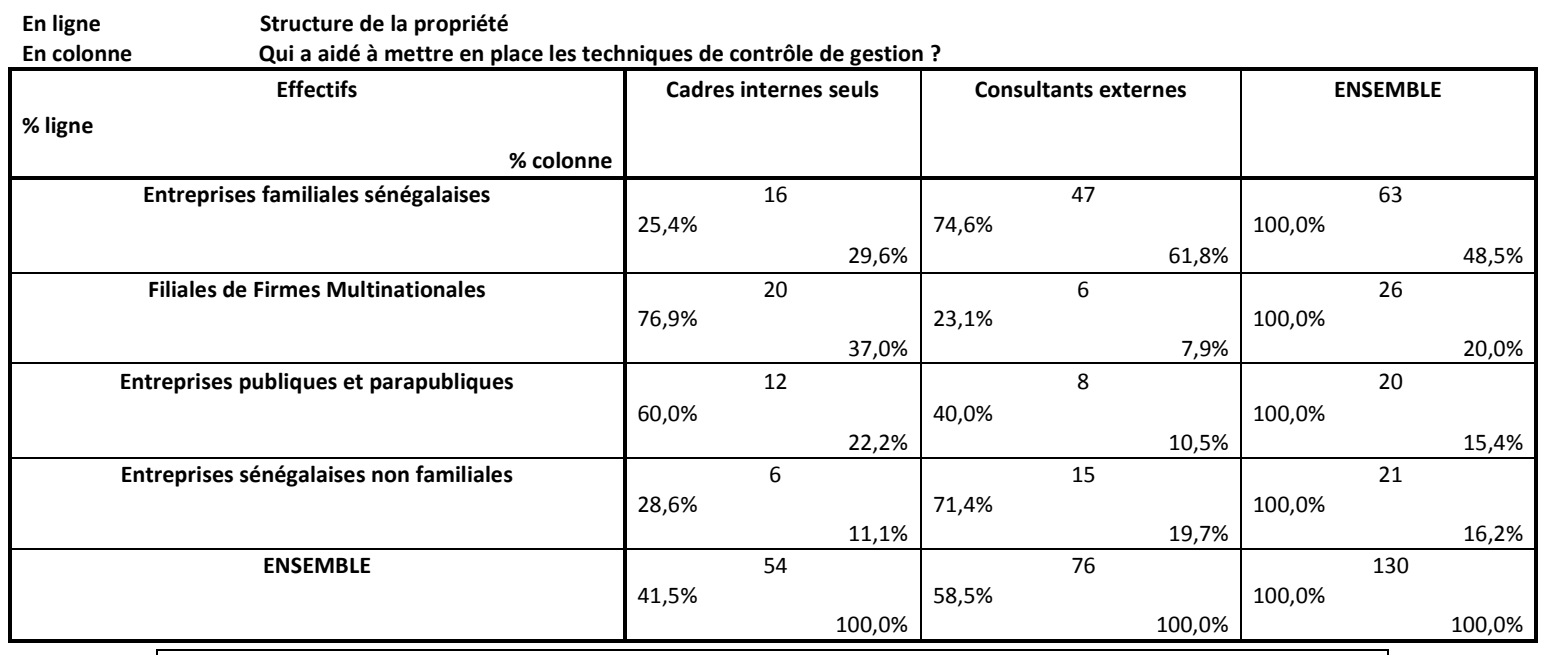

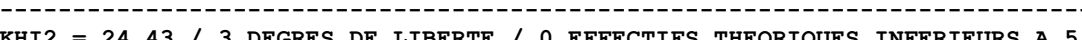

PROBA $($ KHI2 > 24.43) $=0.000 / \mathrm{V} . \mathrm{TEST}=4.10$ 